\title{
Demolición y clausura
}

Nuestra realidad moderna ha desmantelado el mito de la eternidad. Los procesos constructivos actuales tienden a la configuración orgánica; sus elementos tienen una duración determinada, y están irremediablemente sujetos a su obsolescencia física o cultural, una pudrición necesaria para mantener la industria activa. La aceleración de estos procesos replantea cómo la vida en la ciudad, naturalmente ininterrumpida, puede relacionarse con su propia construcción: procedimientos múltiples, entrecortados, paralizantes, velados.

Palabras clave: Arquitectura - teoria, demolición urbana, construcciones ensanches, ruinas, renovación urbana.

The modern world has dismantled the myth of eternity. Today's building processes tend towards the organic; the materials have a limited lifetime and are subject to unavoidable physical or cultural obsolescence, their decay necessary to keep industry active. The speeding up of these processes raises the question, how does life in the city, which. is naturally continuous, relate to its own construction, to procedures that are multiple, intermittent, paralyzing, bidden? Key words: Architecture - theory, urban demolition, constructions,
street-widening, ruins, urban renewal.
Es difícil evitar el sobresalto provocado por una explosión, y cuando va acompañado del espectáculo del desmoronamiento de un edificio, a esa primera emoción se agrega un goce íntimo: el de haberse librado del pasado. Cabe aclarar que, para no entrar en el plano de lo sublime, me refiero a la demolición de edificios a objeto de despejar terreno para dar inicio a una construcción. Es inevitable considerar que las guerras urbanas del siglo $\mathrm{XX}$ produjeron muchas víctimas, pero también escombros, y después de una guerra, los grandes empresarios de la construcción están tan prestos a prestar ayuda, que hace sospechar que algo tuvieron que ver con los bombardeos. Pero el tema que convoca, más allá de cualquier placer íntimo y consideraciones económicas y políticas, es el proceso proyectual contemporáneo.

El interés puesto en el tema de la demolición resulta de los enfoques, ideas y realizaciones nacidas de la angustia por la deflagración de un estatuto que se creía unitario. Desde los primeros años de la segunda post guerra la arquitectura moderna se vio obligada a revisar su plataforma conceptual y sus metodologías, y entre el optimismo y la desilusión, fluyó un rico andamiaje de propuestas que ha resucitado luego del agotamiento postmoderno. Muchas de aquellas ideas habían sufrido una condena que se amparaba tras la ideología que se ocupó de moralizar acerca de lo que debía ser. Así, mientras la nostalgia historicista, las raíces nacionales y populares y la banalidad desplegaban sus celebrados discursos, otras arquitecturas debieron guardar silencio. Claude Schnaidt sentenciaba sobre éstas: "Tales visiones son tranquilizadoras para muchos arquitectos que, alentados por tanta tecnología y por tanta confianza en el futuro, se sienten seguros y justificados en su abdicación social y política" (Frampton, 1981). Pero dicha abdicación social y política fue una anticipación de las condiciones generales de la sociedad contemporánea.

\section{La demolición}

Antes de construir dentro de la ciudad hay que demoler algo. No sucede lo mismo en las periferias urbanas o en la colonización nuevos territorios, situaciones éstas muy frecuentes en la arquitectura moderna. Las razones de esta acción demoledora son de diverso tipo y sin duda, los escombros se llevan consigo algún recuerdo personal o colectivo. También la demolición confirma que se es moderno (en el registro del imaginario colectivo) y por ello, cuando se realiza con gran estrépito e instantáneamente, produce el goce íntimo de quien abriga la esperanza de un futuro mejor y borra amargos pasados. En buena medida, la primera acción productiva del arquitecto es destruir arquitectura. Ocurre con la producción en general: para producir leña o un mueble hay que destruir un bosque.

Tiene interés volver los pasos sobre el significado de esta acción, debido a que la arquitectura, como decía Argan, se sobreentiende como arte metépsico, "que crea y no representa, a diferencia de la pintura y la escultura, que son artes miméticas" (Argan, 1969). Así, la arquitectura se representa a sí misma y se almacena como historia, porque la creación arquitectónica pura no existe, sino que se apoya en su propia experiencia; de otro modo no se podría identificar un hecho como arquitectónico más allá de su capacidad de albergar actividades humanas, y esto no ocurre siempre.

Por estas razones, la arquitectura en la ciudad carga consigo la demolición de su pasado construido para construirse. Esto ocurrió durante siglos en Roma, en la Edad Media, en las ciudades del Renacimiento. La Roma imperial se construyó con piezas múltiples, como un bricolage, al decir de Colin Rowe: “...lo físico $y$ lo político de Roma proporcionan lo que es tal vez el ejemplo más gráfico de tejidos de colisión y deshechos intersticiales..." (Rowe y Koetter, 1981). En efecto, desde la construcción del Forum Julium -el año 54 a.C.-, el de Augusto, el Transitorium, el de Nerva, hasta el de Trajano -construido entre el 112 y 113 d.C.- transcurrieron 159 años, con arcos triunfales, mercados, templos y basílicas. La fiebre constructora fue, sin duda, muy alta. Mientras tanto, la civitas romana continuaba consumiendo el tiempo deambulando por los foros sorteando escombros y aparejos, no tanto por carencia de previsión, sino porque el proceso estaba naturalizado. En realidad ese bricolage era un híbrido compuesto de fragmentos de otros edificios, que se demolían parcialmente o se adosaban. La Edad Media fue testigo de este continuum de apropiaciones, superposiciones y adiciones que los transeúntes vivían con naturalidad, porque formaba parte de la vida urbana.

Relataba el abate Suger acerca de la reconstrucción de St. Denis a mediados del siglo X: “...después de haber extraído de las torres $y$ del techo que corría transversalmente entre ellas cimientos materiales bastante sólidos y de haber puesto los cimientos espirituales aún más sólidos... preocupados en primer lugar de que la parte vieja y la nueva se unieran sin desentonar ni contrastar, nos ocupábamos de dónde podríamos procurarnos columnas de mármol... A fuerza de pensarlo y repensarlo no quedaba otra solución que hacerlas 
Haussmann como "artista

demóvedor". Tomado de

iudad. Ediciones Gustavo Gili,

México D.F., 1978, p.56

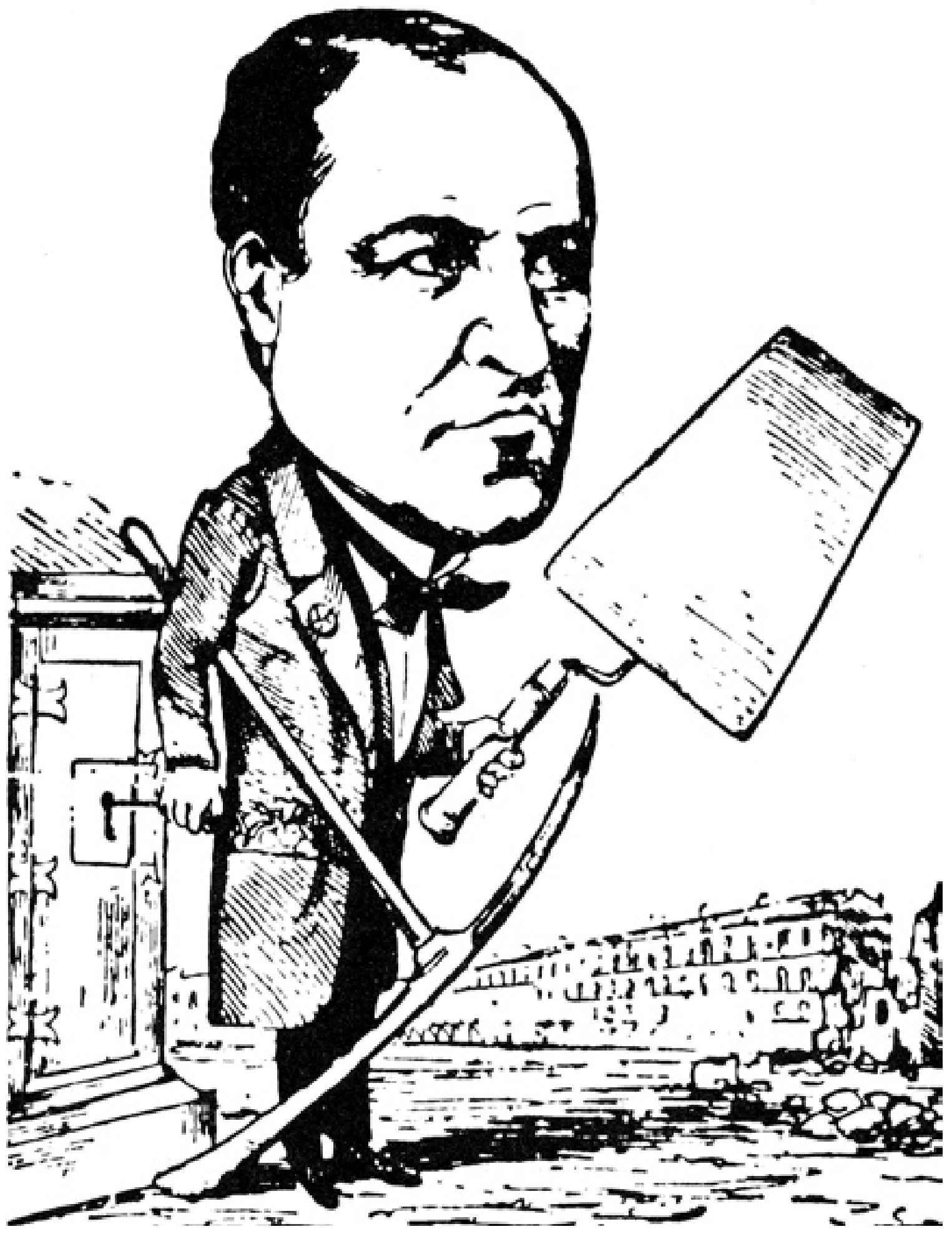


venir de Roma a través de una flota bien protegida, $y$ de alli a través de La Mancha y los meandros del Sena, con gran cantidad de dinero de los amigos y hasta alquilando las naves a los enemigos sarracenos" (Patteta, 1984). Es decir, demolición y rapiña para nueva arquitectura.

Pero el momento estelar de las demoliciones urbanas fue el siglo XIX, cuando la modernización celebra su triunfo escribiendo un palimpsesto, no tanto por falta de papel, sino porque había que borrar las huellas de un pasado insalubre, hacinado, pestilente, promiscuo, oscuro, húmedo, envilecedor, que no permitía el despliegue del progreso y sus manifestaciones urbano arquitectónicas. Así, los ensanches, los boulevares, los parques urbanos, las cloacas y los transportes subterráneos comenzaron a aparecer con el primer acto sublime de la demolición: el pasado fue borrado con pico y pala, y sobre el plano despejado se volvió a escribir un nuevo texto.

Este proceso se acelera en el siglo XX cuando las edificaciones tienen menor duración, entre otras razones, por su propia condición moderna. En efecto, en este siglo y el pasado, la mayoría de los edificios construidos con el empleo de las tecnologías proporcionadas por su propio tiempo están condenados a sufrir el veloz envejecimiento de sus componentes constructivos, porque la modernidad fundó una de sus bases sobre la innovación tecnológica que, por su propia naturaleza, se renueva continuamente y en consecuencia hace menos duradera la vida de los edificios que la alberga. Es sorprendente que cuando éstos envejecen no lo hacen con la dignidad de los antiguos. La ruina moderna, a diferencia de otras, se presenta como despojo decadente de una civilización fundada en el desvanecimiento: un edificio antiguo sin uso y con fragmentos desparramados en el suelo es un bello y nostálgico monumento; un edificio moderno con placas de cielorraso caídas es simplemente un deplorable abandono. El Baudelaire de: "La modernidad es lo transitorio, lo fugitivo, lo contingente" se manifiesta en la industria intrínsecamente, porque debe modificar continuamente sus productos, mejorarlos, aplicar nuevos conceptos, nuevos materiales, nuevas prestaciones, nuevas economías y para ello cambia sus líneas de producción y sus máquinas. De este modo, los materiales modernos resultan menos duraderos, tienen fecha de vencimiento, son como materia orgánica. Así, disponer de un mismo material industrial o mecanismo producido hace un par de décadas es una tarea en extremo difícil porque obliga a reponer la industria que lo produjo.
La arquitectura moderna no es ajena a este problema, y por ello se podría aventurar que es más sencillo reproducir el fuste de una columna del Partenón que un perfil de acero standard de las ventanas de la Bauhaus de Dessau, que Crittall Windows Ltd. realizó en 1926. En su restauración de 1976 se hicieron de aluminio, pese a que la fábrica Crittall Windows todavía está en funcionamiento (Blake, 1990).

Razonablemente, un edificio contemporáneo realizado con nuevas técnicas y materiales nuevos tiene una fecha de vencimiento de cincuenta años. Asumiendo este destino, es evidente que los estatutos clásicos de la arquitectura deben ser revisados, porque la eternidad es un mito brutalmente derribado por una realidad que no sólo es mercantil, sino intrínsecamente moderna. No sin razón, ante el debate acerca de la conservación del Zonestraal de Duiker, algunos opinaban que debía dejarse como una ruina, abandonarse y dejar que lo devorara la maleza (Reinink, 1990). También, más allá de los esfuerzos de Gropius y la mayoría de los arquitectos para evitar la demolición de los almacenes Schocken de Stuttgart, en 1960, Louise Meldelsohn -viuda del autor- aceptaba su destino declarando: "Cuando un edificio ba sido acabado Eric terminaba con él. Su espiritu siempre aspiraba a cosas por venir". El objetivo de la ciudad de Stuttgart en los años sesenta no era la preservación o la conservación sino la progresión. Un especialista en conservación, Lars Scharnholz, comentaba frente a este caso: "Una característica del Movimiento Moderno es que la preservación del contexto bistórico arquitectónico y tratamiento sustentable de los edificios existentes son considerados menos importantes que su evolución".

Paradójicamente, la obra de Mies van der Rohe es una de las más conservadas de la arquitectura moderna: las casas Lange y Esters en Krefeld; los Promontory Apartment, el conjunto de Lake Shore Drive, la casa Farnsworth, y el Pabellón de Barcelona, así como las permanentes restauraciones del campus del IIT (cuyos edificios se están cayendo a pedazos). La restauración de estas piezas maestras obligó a invertir sumas considerables de dinero, pero: "Mientras se trataba de resolver la complejidad tecnológica de estas restauraciones, la discusión sobre los problemas de la preservación se mantenían ausentes" (Scharnholz, 1999).

En la postguerra, Peter Smithson declaraba: "La planificación es un problema de 'andar' más que de partir de una página en blanco. Nosotros aceptamos como un acto fijo lo que una generación bace con mucho esfuerzo. Debemos seleccionar sólo los puntos con mayor significado sobre la totalidad de la estructura urbana, más que bacer frente a una reorganización ideal de la totalidad. Nuestro deseo lógico y estético actual no es construir castillos en el aire sino una suerte de nuevo realismo y nueva objetividad: la consecuencia de nuestra acción en una situación dada" (Nitschke, 1965). Otro connotado representante del ICA, Lawrence Alloway, escribía en 1959: "Las ciudades son, citando a Fobn Rannells, la acumulación de las actividades de la gente, y éstas cambian con más rapidez que los edificios o las ideas de los arquitectos. Louis Sullivan ...dijo que [la arquitectura es el] 'drama de crear cosas que van al olvido'. No bay lugar donde esto resulte más visible que en la poblada y sólida ciudad y en ningún sitio tienen menos posibilidades de permanecer intactos los principios formales permanentes. El pasado, presente y el futuro ...se trasladan en confusa configuración. Los arquitectos nunca pueden conseguir y mantener el control de todos los factores de una ciudad que bay en las dimensiones de las formas apedazadas, en expansión y en desarrollo" (Alloway, 1959).

De estas citas se desprende la búsqueda de una estética del cambio. Cedric Price, en el edificio Inter-Action instaló a la obsolescencia como estética. Había previsto instrucciones para su demolición debido a que el Ayuntamiento había arrendado el terreno por 27 años y de hecho, se demolió en el año 2001. Para ello la estructura, los cerramientos y las instalaciones conformaban un sistema genérico cuyos atributos formales daban señales industriales inequívocas. Decía Price: "Podremos reconocer más fácilmente los cinco estados del tiempo artificial (uso, abuso, re-uso, desuso y rechazo) si concedemos la misma importancia a los intervalos temporales correspondientes a la construcción y la demolición (duración) con el propósito de introducir en el proceso de proyecto factores como el tiempo, la transformación y la reubicación temporal ... La flexibilidad constructiva, o su alternativa, la obsolescencia planificada, sólo pueden conseguirse satisfactoriamente si incluimos el factor temporal como parámetro clave dentro del proceso completo del diseño" (Price, 1996). Cabe destacar la admirable previsionalidad inglesa ante nuestro mientras tanto latinoamericano, que podría eternizar cualquier construcción provisional. Dejando en suspenso intimidades de nuestra cultura, la previsión proyectual de Price determinó en buena medida los aspectos estético - formales de la edificación. Esto fue corriente durante los años sesenta y setenta del viejo siglo pasado; desde el Fun Palace de Price al Centro Pompidou de Piano \& Rogers, la imagen de lo transitorio y lo flexible programado construyó una estética fundada en la técnica, al decir de Ezio Bonfanti, una emblemática tecnológica que trascendió sus propósitos iniciales (Bonfanti, 1969). En efecto, los edificios para durar 
eternamente -dentro de la paradoja secular de nuestra institución arquitectónica- semejaban a construcciones fabriles y ferroviarias. De esto dan cuenta las proposiciones de Cedric Price, de Archigram, de Yona Friedman, de Constant, cuyos argumentos se repiten hoy como si fuesen nuevos: el riesgo que amenaza su credibilidad hoy es, como ocurrió antes, que cuanto más se aleje del mundo fáctico, más inofensiva resulta a su propia institución.

Pero en ese debate, otras voces se han propuesto algo más radical. Martin Pawley adelantaba: "Los esfuerzos de Habraken para estabilizar el mundo en constante evolución de los sistemas de sostenimiento, están condenados antes de baberse iniciado. En este contexto, el cambio no puede ser detenido -aunque éste sea el sueño desesperado de los preservacionistas-. Todo lo que puede hacerse es dotar al espacio bumano de mecanismos capaces de absorber la evidencia del tiempo y del cambio, a fin de mitigar el horror al cambio mismo. Incorporando en cada configuración sucesiva los elementos de todas las que la precedieron, se podría separar el cambio de la destrucción y la pérdida, y conseguir de esta forma un continuum en el campo privado que está aún, en cierta medida, legal y económicamente protegido" (Pawley, 1975). Así, absorbiendo la evidencia del tiempo e incorporando los elementos de todas las configuraciones precedentes, se podrá superar la condición de emblemática técnica que caracteriza a la estética del cambio.

Por ello, la plataforma de la demolición no habla sólo de arquitecturas transformables, sino de operaciones de intervención proyectual que actúan sobre la materia dada que a su vez sabe que será transformada. La paradoja se podría resolver si el proyecto albergara la demolición $\mathrm{y}$ de este modo incorporara un proceso de mutaciones, de transformaciones progresivas, sin solución de continuidad, como ocurre con la ciudad en general. El híbrido resultante de esta proyectación no está demasiado alejado de una condición general de la cultura.

\section{La clausura}

Estas reflexiones tienen su origen en otra paradoja: la vida urbana tiene continuidad, a la que se opone su arquitectura, que es discontinua, celebratoria de acontecimientos aislados, fijos e inmutables. Esto lo decía Yona Friedman en los años sesenta. Para esta celebración, una obra en construcción es protegida, no sólo para seguridad de los transeúntes; también como obra que promete conmover ante su descubrimiento. Es una ideología inaugurada en el Renacimiento: el autor devela, corre el velo que ocultaba su proceso creativo y produce el primer shock ante la mirada atónita y regocijada del mecenas y sus amistades. Los hechos arquitectónicos y artísticos eran concebidos como criaturas humanas: obras escultóricas, frescos y edificios guardaban celosamente su gestación y se develan al público sólo acabadas, como surgidas de un solo impulso creador. Es por estas razones entre otras, que durante el proceso productivo de la obra ésta guarda su secreto, se oculta con vallas y lienzos aguardando por el milagro de la creación humana clausurando un fragmento de ciudad que bloquea y restringe el fluir de los transeúntes, obstaculiza el tránsito de vehículos, impide la circulación monetaria del comercio, obliga desvíos desorientadores e impide que la obra misma forme parte del espectáculo urbano. Con el expediente de obreros en la vía, hombres trabajando, disculpen las molestias, obra en construcción resuelven, más que la incomodidad, la apropiación del espacio urbano con la promesa de un mejor servicio, casi siempre albergado en arquitectura. Mientras esto sucede, la arquitectura agazapada adquiere forma, se gesta, hasta que finalmente se devela. El transeúnte -antes que el arquitecto- se rinde ante esta admirable manifestación de progreso. Escribía Walter Benjamin sobre París del siglo XIX: "La institución del señorío mundano y espiritual de la burguesía encuentra su apoteosis en el manejo de las arterias urbanas. Estas quedaban tapadas con una lona hasta su terminación y se las descubría como a un monumento" (Benjamin, 1972).

Para evitar la histeria metropolitana es hora de acoger los ruidos y polvaredas de las demoliciones - construcciones como parte de nuestra vida urbana y resolver las discontinuidades de una obra atravesándola con el uso de las instalaciones de faena como programa urbano. De este modo, las vallas cobran espesor, son dispositivos, contienen actividades, permiten el tránsito y nada se interrumpe. En este fantástico espectáculo futurista, la circulación monetaria tampoco se interrumpe: el comercio continúa funcionando, el flaneur contemporáneo también.

La demolición que pretendía proporcionar continuidad al ritmo urbano es seguida -como un fractal- con la continuidad urbana durante el proceso mismo de demolición - construcción, con el aprovechamiento del utilaje de protección para proporcionar nuevas actividades. El resultado de esto podría semejarse a las llamadas utopías tecnológicas porque dicho utilaje está constituido por andamios y estructuras metálicas de gran versatilidad, por su propia condición transitoria. Así, la transitoriedad y la mutación se hacen presentes como obra de infraestructura, a la vez que terminada esta operación se pone al descubierto la obra que ocultaba. Sin duda esta sucesión de obras, de obras dentro de obras, en una endemoniada continuidad de máquinas, ruidos y polvo, no es otra cosa que la aceleración del ritmo metropolitano.

Arribamos finalmente a la noción de continuidad urbana, que en realidad es una sucesión de eventos. Éstos, en términos arquitectónicos, pueden localizarse en un punto de cierto interés: una obra contemporánea se sostiene sin cambios sustantivos durante 50 años; con cambios necesarios, 20 años; con cambios imprescindibles, apenas el arquitecto entrega a sus clientes la obra concluida; luego, las instalaciones de infraestructura se mantienen durante 2 años, que corresponde a la duración de la obra, pero podrían convertirse en infraestructuras con programas urbanos activos y rentables. Ahora bien, como la construcción de las instalaciones de infraestructura demora un par de días, entonces ellas a su vez podrían albergar programas para evitar la clausura urbana correspondiente; ergo, la construcción de las obras de infraestructura para la construcción de un edificio es el verdadero evento, un espectáculo. Sólo en ese momento un sector de la ciudad se detiene; por esta razón, glosando a Pirandello, los personajes están buscando a un autor. ARQ

Bibliografía: Alloway, Lawrence; Architectural Design, enero de 1959. / Argan, Giulio Carlo; Proyecto y destino. Universidad Central de Venezuela, Caracas, 1969, p. 71. / Benjamin, Walter; Iluminaciones II. Ed. Taurus, Madrid, 1972, p. 187 / Blake, David; "Windows, Crittall and the Modern Movement". do.co.mo.mo. First International Conference, do.co.mo.mo international, París, 1990, p. 76-79. / Bonfanti, Ezio; "Emblemática de la técnica”. Cuadernos Summa-Nueva Visión $\mathrm{N}^{\circ}$ 43, 1969, p. 14-31. / Frampton, Kenneth; Historia crítica de la arquitectura moderna. Ed. Gustavo Gili, Barcelona, 1981, p. 290. / Nitschke, Günter; "Cities stasis or process". The Pedestrian in the City, Ed. David Lewis, Elek Books, Londres, 1965, p. 165. / Patetta, Luciano; Historia de la arquitectura, antología crítica. Ed. Hermann Blume, Madrid, 1984, p. 96. / Pawley, Martin; "La casa del tiempo". El significado en arquitectura, ed. Charles Jencks y George Baird, Ed. Hermann Blume, Madrid, 1975, pp. 152. / Price, Cedric; Arcbitect's fournal. sept. 5, 1996, p. 38. / Reinink, Wessel; "Controversy between functionalism and restoration: keep Zonnestraal for eternity as a ruin". do.co.mo.mo. First International Conference, 1990, p. 50. / Rowe, Colin y Koetter, Fred; Ciudad collage. Ed. Gustavo Gili, Barcelona, 1981, p. 105. / Scharnholz, Lars; "Preserving the memory". do.co.mo.mo fournal $\mathrm{n}^{\circ}$ 21, do.co.mo.mo international, París, 1999, p. 55. 